13,01

\title{
Оценка характеристик однофотонного детектора в зависимости от параметров сверхпроводящей пленки $\mathrm{W}_{x} \mathrm{Si}_{1-x}$
}

\author{
() С.Ю. Хыдырова, И.А. Степанов, Д.Д. Васильев, К.М. Моисеев \\ Московский государственный технический университет им. Н.Э. Баумана, \\ Москва, Россия \\ E-mail: hydyrova.selbi@yandex.ru
}

Поступила в Редакцию 9 апреля 2021 г.

В окончательной редакции 9 апреля 2021 г.

Принята к публикации 19 апреля 2021 г.

\begin{abstract}
Проведен расчет эффективности поглощения, пороговой длины волны и длительности импульса напряжения сверхпроводникового однофотонного детектора в зависимости от поверхностного сопротивления $R_{s}$ и критической температуры $T_{c}$ пленки $\mathrm{W}_{x} \mathrm{Si}_{1-x}$. Рассчитанные зависимости характеристик детектора для пленок $\mathrm{W}_{x} \mathrm{Si}_{1-x}$ показывают, что пленки с $T_{c}>3.5 \mathrm{~K}$ и $300<R_{s}<420 \Omega / \mathrm{sq}$ обеспечат $\eta_{a b s} \approx 15 \ldots 20 \%$, $\eta_{\mathrm{IDE}}=100 \%$ при длине волны излучения $1550 \mathrm{~nm}$ и длительность импульса напряжения детектора $\tau<40 \mathrm{~ns}$, что соответствует возможной скорости счета детектора $C R_{\max }=25 \mathrm{MHz}$.
\end{abstract}

Ключевые слова: однофотонный детектор на сверхпроводящей нанопроволоке (SNSPD), эффективность детектора, скорость счета детектора, тонкая пленка $\mathrm{W}_{x} \mathrm{~S}_{(i-x)}$, поверхностное сопротивление, критическая температура, сверхпроводимость, чувствительный элемент.

DOI: 10.21883/FTT.2021.09.51268.29H

\section{1. Введение}

Сверхпроводниковые однофотонные детекторы востребованы в системах дальней космической связи, ЛИДАРах, квантовых вычислениях и оптической томографии [1]. Одним из наиболее перспективных материалов чувствительного элемента является $\mathrm{W}_{x} \mathrm{Si}_{1-x}$, эффективность детекторов на основе которого достигает 93\% [2]. Параметры сверхпроводящей пленки чувствительного элемента значительно влияют на выходные характеристики однофотонного детектора на сверхпроводящей нанопроволоке (SNSPD). Целью настоящей работы является выявление параметров пленок $\mathrm{W}_{x} \mathrm{Si}_{1-x}$, которые обеспечат как высокую квантовую эффективность, так и малое время восстановления SNSPD. Для этого проводится расчет характеристик SNSPD на основании экспериментальных значений критической температуры $T_{c}$ и поверхностного сопротивления $R_{s}$ наших пленок $\mathrm{W}_{x} \mathrm{Si}_{1-x}$.

\section{2. Формирование пленок $\mathrm{W}_{x} \mathrm{Si}_{1-x}$ чувствительного элемента детектора}

В лаборатории „Вакуумные технологии и объекты наноинженерии“ кафедры „Электронные технологии в машиностроении“ ультратонкие пленки $\mathrm{W}_{x} \mathrm{Si}_{1-x}$ формируются методом совместного магнетронного распыления из двух источников. При отработке режимов формирования пленок с требуемыми свойствами варьировались следующие параметры процесса: поток аргона $Q_{\text {Ar }}$ от 1.04 до $3.381 / \mathrm{h}$, рабочее давление в камере $P_{\text {oper }}$ от $2.0 \cdot 10^{-3}$ до $2.8 \cdot 10^{-3}$ mbar и время осаждения плен- ки; контролировалось значение остаточного давления в вакуумной камере $P_{\text {res }}$ от $0.5 \cdot 10^{-3}$ до $3.0 \cdot 10^{-3} \mathrm{mbar}$. Объемная доля вольфрама в пленке от 65 до 85\% достигалась варьированием мощностей магнетронов. Пленки формировали на подложках $\mathrm{Si}, \mathrm{SiO}_{2}$ и сапфировой. Толщина сформированных пленок $d$ оценивалась косвенно по времени осаждения и измеренной кварцевым микровзвешиванием скорости осаждения и составляет от 5.6 до $12.9 \mathrm{~nm}$. Поверхностное сопротивление пленок $R_{s}$ измерялось четырехзондовым методом и составляет от 191 до $587 \Omega /$ sq. Критическая температура пленок $T_{c}$ составляет от 2.62 до $4.67 \mathrm{~K}$; измерения проводились с использованием сосуда Дьюара и откачиваемой вакуумным насосом вставки, позволяющей охладить образец до температуры $1.7 \mathrm{~K}$.

\section{3. Расчет эффективности поглощения пленки}

Эффективность детектирования определяется эффективностью поглощения и эффективностью генерации импульса (внутренней эффективностью детектирования).

Для сплошной тонкой металлической пленки с высокой проводимостью эффективность поглощения $\eta_{\mathrm{ABS}}$ может быть определена как [3]:

$$
\eta_{\mathrm{ABS}}=\frac{4 Z_{0} R_{S}}{\left[Z_{0}+\left(n_{\mathrm{sub}}+1\right) R_{S}\right]^{2}}
$$

где $Z_{0}=377 \Omega$ - импеданс вакуума, $R_{s}$ - поверхностное сопротивление пленки, $n_{\text {sub }}-$ коэффициент преломления подложки. Наличие промежутков между 


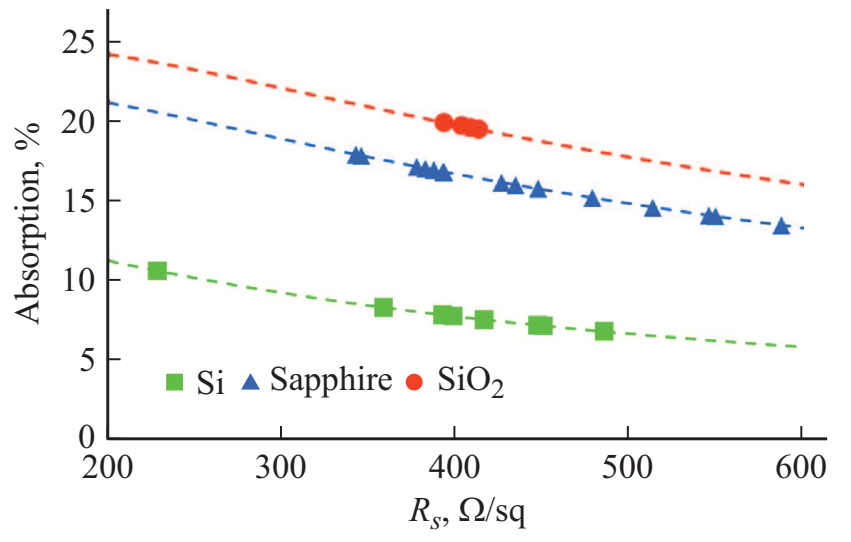

Рис. 1. Зависимость эффективности поглощения пленки $\mathrm{W}_{x} \mathrm{Si}_{1-x}$ на различных подложках от ее поверхностного сопротивления $R_{s}$. Квадраты, треугольники и кружки $(\boldsymbol{\bullet}, \boldsymbol{\Delta}$ и $\bullet)-$ экспериментальные значения $R_{s}$ пленок $\mathrm{W}_{x} \mathrm{Si}_{1-x}$, сформированных на подложках из $\mathrm{Si}$, сапфира и $\mathrm{SiO}_{2}$ соответственно.

полосками меандра снижает эффективность поглощения пропорционально коэффициенту заполнения меандpa $f[4]$.

Для расчета эффективности поглощения чувствительного элемента на основе пленок $\mathrm{W}_{x} \mathrm{Si}_{1-x}$ коэффициент заполнения меандра примем равным $f=0.6$; коэффициенты преломления материалов подложек кремния, сапфира и диоксида кремния при длине волны $1550 \mathrm{~nm}$ составляют соответственно $n_{\mathrm{Si}}=3.475 ; n_{\text {Sapphire }}=1.745$; $n_{\mathrm{SiO}_{2}}=1.444$. Результаты расчета эффективности поглощения приведены на рис. 1.

Наибольшее рассчитанное значение эффективности поглощения соответствует пленкам $\mathrm{W}_{x} \mathrm{Si}_{1-x}$ на подложках из $\mathrm{SiO}_{2}$ и достигает $\sim 20 \%$ при поверхностном сопротивлении пленки $393 \Omega / \mathrm{sq}$.

\section{4. Расчет пороговой длины волны детектора}

Внутренняя эффективность детектирования $\eta_{\mathrm{IDE}}$ определяется длиной волны излучения, геометрией меандра и параметрами пленки. Согласно модели горячего пятна, максимальное значение ширины полоски, при которой сохранится наибольшая эффективность детектирования, в случае полного подавления параметра порядка в горячем пятне можно оценить на основании размера горячего пятна и отношения тока смещения к критическому. Для детектирования по механизму горячего пятна при полном подавлении параметра порядка необходимо, чтобы отношение тока смещения к критическому току удовлетворяло следующему выражению [5]:

$$
\frac{I_{b}}{I_{c}} \geq 1-\frac{2 R}{w}
$$

где $w$ - ширина нанопроволоки, $R$ - радиус горячего пятна. Диаметр горячего пятна $2 R$ равен длине термали- зации $L_{t h}=\sqrt{D \tau_{t h}}[5]$, где $D-$ коэффициент диффузии электронов, $\tau_{t h}$ - время термализации квазичастиц. Для пленок $\mathrm{W}_{x} \mathrm{Si}_{1-x} D=0.58 \mathrm{~cm}^{2} / \mathrm{s}[6], \tau_{t h}=14 \mathrm{ps}$ [6], тогда диаметр горячего пятна составляет $2 R \sim 28.5 \mathrm{~nm}$. Исходя из приведенного выражения, в случае полного подавления параметра порядка, для нанопроволоки шириной $w=100 \mathrm{~nm}$ детектирование будет обеспечиваться при отношении тока смещения к критическому $I_{b} / I_{c} \gtrsim 0.72$.

Для полного подавления сверхпроводимости в горячем пятне необходимо, чтобы энергия поглощенного фотона превышала требуемую минимальную энергию $E_{p h}[5]$ :

$$
E_{p h}=d \pi L_{t h}^{2}\left(\frac{N_{\mathrm{F}}(0) \Delta^{2}}{2}+\frac{2 \pi^{2} N_{\mathrm{F}}(0)\left(k_{b} T_{c}\right)^{2}}{3\left(1+\frac{C_{p h}\left(T_{c}\right)}{C_{e}\left(T_{c}\right)}\right)}\right),
$$

где $d-$ толщина пленки чувствительного элемента; $N_{\mathrm{F}}(0)=1 /\left(R_{s} D d q^{2}\right)$ - плотность электронных состояний на уровне Ферми при $0 \mathrm{~K}[4] ; q$ - заряд электрона; $\Delta=1.764 k_{b} T_{c} /\left(1-\left(T / T_{c}\right)^{4}\right)-$ энергетическая щель [7]; $T_{c}$ - критическая температура пленки; $T$ - рабочая температура детектора; $C_{p h} / C_{e}-$ отношение фононной и электронной теплоемкостей пленки при $T_{c}$ [6]. Учитывая, что длина волны излучения определяется как $\lambda_{p h}=h c / E_{p h}[8]$, можно рассчитать максимальную длину волны фотона, при поглощении которого, в случае соблюдения описанных выше требований по току смещения для заданной ширины нанопроволоки, в образовавшемся горячем пятне будет полностью подавляться параметр порядка и обеспечится детектирование фотона

$$
\lambda_{c}=\frac{h c R_{s} q^{2}}{\pi \tau_{t h}\left(k_{B} T_{c}\right)^{2}\left(\frac{1.764^{2}\left(1-\frac{T}{T_{c}}\right)}{2}+\frac{2 \pi^{2}}{3\left(1+\frac{C_{p h}\left(T_{c}\right)}{C_{e}\left(T_{c}\right)}\right)}\right)} .
$$

Поглощенные нанопроволокой фотоны с длиной волны менее $\lambda_{c}$ будут обеспечивать отклик детектора с вероятностью $100 \%$, что соответствует внутренней эффективности $\eta_{\mathrm{IDE}}=100 \%$. Поэтому $\lambda_{c}$ можно считать пороговой длиной волны детектора.

Из полученного для $\lambda_{c}$ выражения, задавшись значением рабочей температуры детектора $T$, а также значениями времени термализации $\tau_{t h}$ и отношения $C_{p h} / C_{e}$ для материала чувствительного элемента, получим зависимость $\lambda_{c}$ от параметров $R_{s}$ и $T_{c}$ сверхпроводящей пленки чувствительного элемента. Принимая $T=0.2 \mathrm{~K}$ и для $\mathrm{W}_{x} \mathrm{Si}_{1-x} \tau_{t h}=14 \mathrm{ps}, C_{p h} / C_{e}=0.18[6]$, а также подставляя вместо $\lambda_{c}$ значение телекоммуникационной длины волны $\lambda_{p h}=1550 \mathrm{~nm}$, мы выразили зависимость значений поверхностного сопротивления $R_{s}$ пленки от ее критической температуры $T_{c}$, при которых полностью подавляется параметр порядка в горячем пятне после поглощения фотона с длиной волны $\lambda_{p h}=1550 \mathrm{~nm}$. Рабочая температура в данном расчете принята равной $T=0.2 \mathrm{~K}$, поскольку уменьшение рабочей температуры 


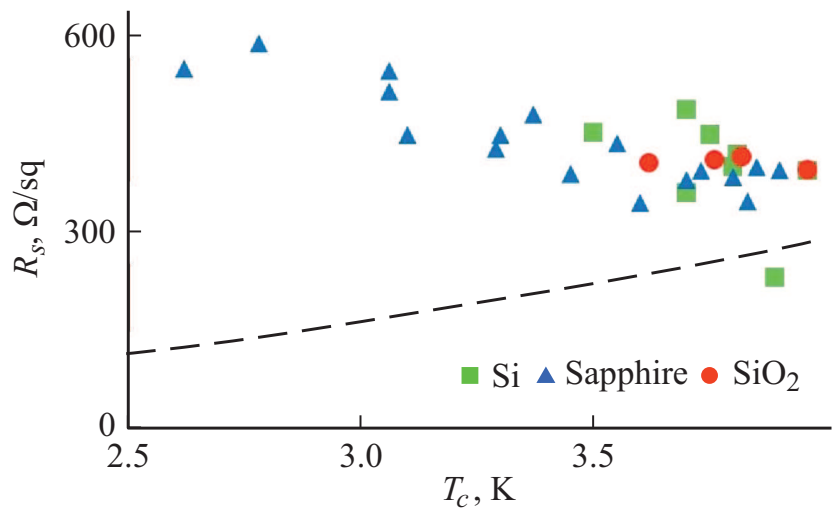

Рис. 2. Зависимость $R_{s}$ от $T_{c}$, при которых обеспечивается $\eta_{\mathrm{IDE}}=100 \%$ для пленок $\mathrm{W}_{x} \mathrm{Si}_{1-x}$ при длине волны $\lambda_{p h}=1550 \mathrm{~nm}$, ширине полоски $w=100 \mathrm{~nm}$ и $I_{b} / I_{c} \gtrsim 0.72$. Квадраты, треугольники и кружки $(\boldsymbol{\bullet}, \boldsymbol{\Delta}$ и $\bullet)-$ экспериментальные значения $R_{s}$ пленок $\mathrm{W}_{x} \mathrm{Si}_{1-x}$, сформированных на подложках из $\mathrm{Si}$, сапфира и $\mathrm{SiO}_{2}$ соответственно.

детектора позволяет снизить число темновых отсчетов [2]. Рассчитанная зависимость $R_{s}\left(T_{c}\right)$ для исследуемых плёнок $\mathrm{W}_{x} \mathrm{Si}_{1-x}$ представлена на рис. 2 .

Область выше кривой на графике рис. 2 соответствует параметрам пленки $\mathrm{W}_{x} \mathrm{Si}_{1-x}$, при которых будет полностью подавляться параметр порядка в горячем пятне после поглощения фотона с длиной волны $1550 \mathrm{~nm}$. Для нанопроволоки шириной $w=100 \mathrm{~nm}$, сформированной на базе пленок с данными параметрами, при отношении тока смещения к критическому $I_{b} / I_{c} \gtrsim 0.72$, детектирование фотонов с длиной волны до $1550 \mathrm{~nm}$ будет обеспечиваться с внутренней эффективностью $\eta_{\mathrm{IDE}}=100 \%$.

\section{5. Расчет длительности импульса напряжения детектора}

Максимально возможная скорость счета детектора $C R_{\max }$ ограничивается длительностью возникающего при детектировании фотона импульса напряжения $\tau$ следующим образом: $C R_{\max }=1 / \tau$ [9]. Длительность импульса напряжения, или время восстановления детектора $\tau$ [9], складывается из времени нарастания $\tau_{\text {rise }}=L_{k} /\left(R_{n}+R_{L}\right) \quad[10]$ и времени спада $\tau_{\text {fall }}=L_{k} / R_{L}[10]$ импульса, где $L_{k}-$ кинетическая индуктивность чувствительного элемента детектора, $R_{n}-$ сопротивление нанопроволоки, возникающее при детектировании фотона, $R_{L}=50 \Omega-$ шунтирующее сопротивление [9]. Поскольку сопротивление $R_{n} \sim 1 \mathrm{k} \Omega$, вклад $\tau_{\text {rise }}$ в длительность импульса напряжения составит порядка $1 \mathrm{~ns}$ [11]. Для приближенной оценки предельной скорости счета детектора можно пренебречь временем нарастания импульса $\tau_{\text {rise, }}$ однако для численной оценки влияния параметров пленки $\left(R_{s}\right.$ и $\left.T_{c}\right)$ следует учитывать как время нарастания, так и время спада импульса.
Кинетическая индуктивность чувствительного элемента детектора определяется как $L_{k}=\mu_{0} \lambda^{2} l /(w d)$ [12], где $\lambda$ - глубина проникновения магнитного поля сверхпроводящей пленки при температуре $T, \mu_{0}-$ магнитная постоянная, а $l, w$ и $d-$ длина, ширина и толщина нанопроволоки соответственно. Температурная зависимость глубины проникновения магнитного поля приближенно описывается эмпирической формулой $\lambda=\lambda_{0} /\left(1-\left(T / T_{c}\right)^{4}\right)^{1 / 2}[13]$, где $\lambda_{0}-$ глубина проникновения магнитного поля при $T=0 \mathrm{~K}$. В [14] $\lambda_{0}$ для грязного предела выражается как $\lambda_{0}=\left[\hbar \rho N / \pi \mu_{0} \Delta_{0}\right]^{0.5}$, где $\rho_{N}$ - удельное сопротивление сверхпроводника, $\Delta_{0}-$ энергетическая щель при $T=0 \mathrm{~K}$. Поскольку $\Delta_{0}=1.764 k_{b} T_{c} \quad[14]$ и $\rho_{N}=R_{s} d$ [14], кинетическую индуктивность чувствительного элемента детектора $L_{k}$ можно выразить через параметры сверхпроводящей пленки $R_{s}$ и $T_{c}$ следующим образом:

$$
L_{k}=\frac{\hbar l R_{s}}{1.764 \pi k_{b} w T_{c}\left[1-\left(\frac{T}{T_{c}}\right)^{4}\right]} .
$$

Приведенные выше зависимости для $\tau, \tau_{\text {rise }}, \tau_{\text {fall }}$ и $L_{k}$ позволяют выразить длительность импульса детектора через параметры $R_{s}$ и $T_{c}$ сверхпроводящей пленки чувствительного элемента

$$
\tau=\frac{\hbar l}{w \pi k_{B}}\left(\frac{1}{R_{L}+R_{n}}+\frac{1}{R_{L}}\right) \frac{R_{s}}{1.764 T_{c}\left[1-\left(\frac{T}{T_{c}}\right)^{4}\right]} .
$$

Для расчета времени восстановления детектора с чувствительным элементом на основе пленок $\mathrm{W}_{x} \mathrm{Si}_{1-x}$ длина и ширина нанопроволоки приняты равными $l=1000 \mu \mathrm{m}$ и $w=100 \mathrm{~nm}$, рабочая температура детектора принята равной, как и в расчете пороговой длины волны детектора, $T=0.2 \mathrm{~K}$; результаты расчета приведены на рис. 3. Кроме того, были рассчитаны значения скорости счета $C R_{\max }$ с учетом обеих составляющих времени восстановления детектора $\tau_{\text {rise }}$ и $\tau_{\text {fall }}$

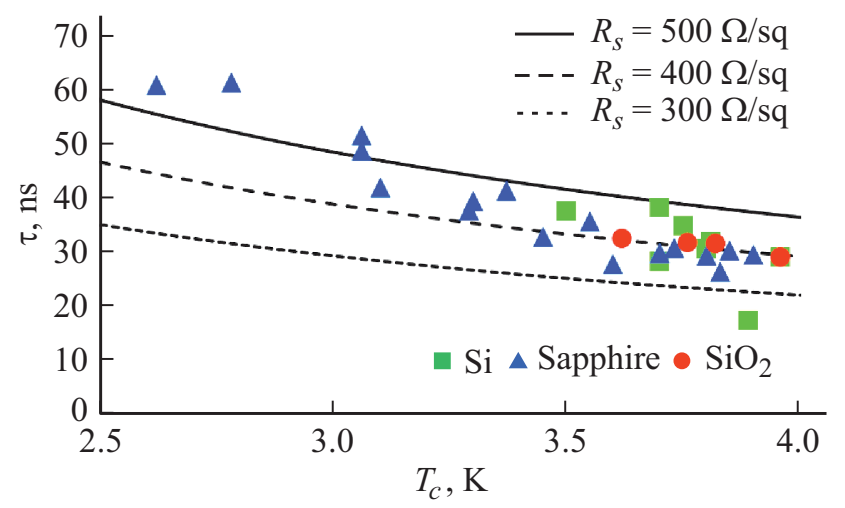

Рис. 3. Зависимость времени восстановления детектора от критической температуры $T_{c}$ пленок $\mathrm{W}_{x} \mathrm{Si}_{1-x}$. Квадраты, треугольники и кружки $(\boldsymbol{\bullet}, \boldsymbol{\Delta}$ и •) - экспериментальные значения $R_{s}$ пленок $\mathrm{W}_{x} \mathrm{Si}_{1-x}$, сформированных на подложках из $\mathrm{Si}$, сапфира и $\mathrm{SiO}_{2}$ соответственно. 
и с учетом только $\tau_{\text {fall }}$; различие результатов точного и приближенного расчета $C R_{\max }$ составило $\sim 1 \mathrm{MHz}$, что подтвердило необходимость точного расчета времени восстановления детектора $\tau$ с учетом $\tau_{\text {rise. }}$

Согласно представленным на рис. 3 результатам расчета, пленки с критической температурой $T_{c}$ более $3.5 \mathrm{~K}$ и поверхностным сопротивлением $R_{s}$ до $450 \Omega / \mathrm{sq}$ обеспечат время восстановления детектора $\tau$ от 20 до $40 \mathrm{~ns}$, что соответствует скорости счета детектора $C R_{\max }$ от 25 до $50 \mathrm{MHz}$, при длине нанопроволоки $l=1000 \mu \mathrm{m}$, ширине $w=100 \mathrm{~nm}$ и рабочей температуре детектора $T=0.2 \mathrm{~K}$.

\section{6. Заключение}

Предложенные в работе выражения позволяют оценить влияние поверхностного сопротивления $R_{S}$ и критической температуры $T_{c}$ сверхпроводящей пленки чувствительного элемента на характеристики детектора. Рассчитанные зависимости характеристик детектора для пленок $\mathrm{W}_{x} \mathrm{Si}_{1-x}$ показывают, что пленки с $T_{c}>3.5 \mathrm{~K}$ и $300<R_{s}<420 \Omega / \mathrm{sq}$ обеспечат эффективность поглощения $\eta_{\mathrm{ABS}} \approx 15 \ldots 20 \%, \eta_{\mathrm{IDE}}=100 \%$ при длине волны излучения $\lambda_{p h}=1550 \mathrm{~nm}$ и время восстановления детектора $\tau$ от 20 до $40 \mathrm{~ns}$ (скорость счета детектора $C R_{\max }$ от 25 до $50 \mathrm{MHz}$ ) при длине нанопроволоки $l=1000 \mu \mathrm{m}$, ширине $w=100 \mathrm{~nm}$ и рабочей температуре $T=0.2 \mathrm{~K}$. На основании полученных результатов и выдвинутых к параметрам пленки требованиий возможно проведение экспресс-оценки пригодности сформированной пленки $\mathrm{W}_{x} \mathrm{Si}_{1-x}$ для изготовления на ее базе однофотонного детектора с высокой квантовой эффективностью и высокой скоростью счета.

\section{Конфликт интересов}

Авторы заявляют, что у них нет конфликта интересов.

\section{Список литературы}

[1] О.В. Минаева, О.В. Окунев, Г.М. Чулкова. Быстродействующий однофотонный детектор на основе тонкой сверхпроводниковой пленки NbN. Прометей, М. (2013). 144 с. ISBN: 978-5-7042-2475-4

[2] F. Marsili, V.B. Verma, J.A. Stern, S. Harrington, A.E. Lita, T. Gerrits, I. Vayshenker, B. Baek, M.D. Shaw, R.P. Mirin, S.W. Nam. Nature Photonics 7, 3, 210 (2013).

DOI: $10.1038 /$ nphoton.2013.13

[3] A. Semenov, B. Günther, U. Böttger, H.-W. Hübers, H. Bartolf, A. Engel, A. Schilling, K. Ilin, M. Siegel, R. Schneider, D. Gerthsen, N.A. Gippius. Phys. Rev. B 80, 5, 054510 (2009). DOI: 10.1103/PhysRevB.80.054510

[4] А.А. Корнеев, Однофотонные детекторы видимого и инфракрасного диапазонов из тонких сверхпроводящих пленок $\mathrm{NbN}$ и $\alpha$-MoSi. Докт. дис. МПГУ, М. (2015).
[5] A. Korneev, A. Semenov, D. Vodolazov, G.N. Gol'tsman, R. Sobolewski. In: Superconductors at the Nanoscale: From Basic Research to Applications / Ed. R. Wördenweber, V. Moshchalkov, S. Bending, F. Tafuri. Walter de Gruyter GmbH, Berlin-Boston (2017). 283 p.

[6] D.Y. Vodolazov. Phys. Rev. Appl. 7, 3, 034014 (2017). DOI: 10.1103/PhysRevApplied.7.034014

[7] М. Тинкхам. Введение в сверхпроводимость. М.: Атомиздат (1979). [M. Tinkham. Introduction to Superconductivity. 2nd ed. McGraw-Hill, Inc., N.Y. (1996) P. 63].

[8] В.Е. Белонучкин, Д.А. Заикин, Ю.М. Ципенюк. Основы физики: квантовая и статистическая физика. Термодинамика/ Под ред. Ю.М. Ципенюка. Физматлит, М.( 2007). 608 с. ISBN 978-5-9221-0754-9

[9] S. Ferrari, C. Schuck, W. Pernice. Nanophotonics 7, 11, 59 (2018). DOI: 10.1515/nanoph-2018-0059

[10] A.J. Kerman, E.A. Dauler, W.E. Keicher, J.K. Yang, K.K. Berggren, G. Gol'tsman, B. Voronov. Appl. Phys. Lett. 88, 11, 111116 (2006). DOI: 10.1063/1.2183810

[11] E.E. Wollman, V.B. Verma, A.E. Lita, W.H. Farr, M.D. Shaw, R.P. Mirin, S.W. Nam. Opt. Express 27, 24, 35279 (2019). DOI: $10.1364 /$ OE.27.035279

[12] D. Li, R. Jiao. Photon. Res. 7, 8, 847-852 (2019). DOI: 10.1364/PRJ.7.000847

[13] A.M. Kadin. Introduction to superconducting circuits. WileyInterscience, N.Y. (1999). P. 32

[14] X. Zhang, A. Engel, Q. Wang, A. Schilling, A. Semenov, M. Sidorova, H.W. Huebers, I. Charaev. K. Ilin, M. Siegel. Phys. Rev. B 94, 17, 174509 (2016). DOI: 10.1103/PhysRevB.94.174509

Редактор Ю.Э. Китаев

Публикация материалов Симпозиума завершена. 